\title{
Spatial analysis and simulation tools for groundwater management: the FREEWAT platform
}

\section{Strumenti di analisi spaziale e simulazione per la gestione delle acque di falda: la piattaforma FREEWAT}

Rudy Rossetto, Giovanna De Filippis, Iacopo Borsi, Laura Foglia, Massimiliano Cannata, Rotman Criollo, Enric Vásquez-Suñé

\begin{abstract}
Riassunto: FREEWAT è un progetto HORIZON 2020 finanziato dalla Comunità Europea relativamente alla call WATER INNOVATION: BOOSTING ITS VALUE FOR EUROPE. Il principale risultato di FREEWAT è lo sviluppo di una piattaforma modellistica open source e di pubblico dominio integrata in GIS per la simulazione della disponibilità e della qualità delle acque sotterranee, con un modulo integrato per la gestione e la pianificazione della risorsa idrica. FREEWAT si propone di promuovere la gestione della risorsa idrica semplificando l'appli-
\end{abstract}

Parole chiave: FREEWAT, GIS, open source, approccio partecipato, gestione delle acque sotterranee.

Keywords: FREEWAT, GIS, open source, participatory approach, groundwater management.

\section{Rudy ROSSETTO 夆”。}

Giovanna DE FILIPPIS

Istituto di Scienze della Vita, Scuola Superiore Sant'Anna, Pisa, Italy

r.rossetto@santannapisa.it

g.defilippis@santannapisa.it

\section{Iacopo BORSI}

TEA SISTEMI S.p.A., Pisa, Italy

iacopo.borsi@tea-group.com

Laura FOGLIA,

Institut für Angewandte Geowissenschaften, Technische Universität Darmstadt, Darmstadt, Germany

lfoglia@ucdavis.edu

\author{
Massimiliano CANNATA \\ Istituto di Scienze della Terra, \\ Scuola Universitaria Professionale della Svizzera Italiana \\ Canobbio, Switzerland \\ massimiliano.cannata@supsi.ch
}

\section{Enric VÁSQUEZ-SUÑÉ}

Rotman CRIOLLO

Instituto de Diagnóstico Ambiental y Estudios del Agua

Consejo Superior de Investigaciones Científicas, Barcelona, Spain

rotman.criollo@idaea.csic.es; enric.vasquez@idaea.csic.es

Ricevuto/Received: 15 September 2017-Accettato/Accepted: 18 September 2017 Pubblicato online/Published online: 30 September 2017

This is an open access article under the CC BY-NC-ND license:

http://creativecommons.org/licenses/by-nc-nd/4.0/

(C) Associazione Acque Sotterranee 2017 cazione della Water Framework Directive e di altre direttive EU legate all'acqua.

A tale scopo, la piattaforma FREEWAT è basata sull'integrazione in QGIS di codici spazialmente distribuiti e fisicamente basati (principalmente appartenenti alla famiglia MODFLOWUSGS), che permettono di analizzare in maniera dettagliata la dinamica delle acque sotterranee, prendendo in considerazione la variabilità spaziale e temporale delle forzanti che controllano il ciclo idrologico. Il tutto avviene in un unico ambiente GIS, dove è possibile gestire e visualizzare ampi set di dati spaziali.

In questo contributo viene fornita una panoramica degli strumenti e dei moduli integrati in FREEWAT per il pre-processamento dei dati e per l'implementazione dei modelli numerici. L'applicabilità della piattaforma FREEWAT è stata dimostrata su 14 casi di studio, nel contesto generale di un approccio partecipato innovativo, che consiste nel coinvolgere tecnici e stakeholder (in primis decisori e responsabili politici) durante le attività modellistiche, creando così un ambiente comune sul tema della gestione della risorsa idrica.

Abstract: FREEWAT is an HORIZON 2020 project financed by the EU Commission under the call WATER INNOVATION: BOOSTING ITS VALUE FOR EUROPE. FREEWAT main result is an open source and public domain GIS-integrated modeling environment for the simulation of groundwater quantity and quality, with an integrated water management and planning module. FREEWAT aims at promoting water resource management by simplifying the application of the Water Framework Directive and other EU water-related Directives. To this scope, the FREEWAT platform results from the integration in the QGIS Desktop of spatially distributed and physically-based codes (mostly belonging to the USGS MODFLOW family), which allow to get a deep insight in groundwater dynamics, taking into account the space and time variability of stresses which control the bydrological cycle. This is attempted in a unique GIS environment, where large spatial datasets can be managed and visualized.

In this paper, a review of the tools/modules integrated in FREEWAT for data pre-processing and model implementation is provided.

FREEWAT applicability was demonstrated through running 14 case studies, in the general framework of an innovative participatory approach, which consists in involving technical staff and relevant stakebolders (in primis policy and decision makers) during modeling activities, thus creating a common environment to enbance science and evidence-based decision making in water resource management. 


\section{Introduzione}

Le decisioni sulla gestione e la pianificazione della risorsa idrica, adottate per applicare le Direttive Europee sull'Acqua (ad es., Water Framework Directive - WFD, Groundwater Directive - GWD), sono spesso effettuate sulla base di bilanci idrologici mediati su periodi temporali di 20/30 anni ed ampie aree geografiche. Detti bilanci non considerano né la variabilità spaziale, né la variabilità temporale delle varie forzanti che controllano gli andamenti annuali ed inter-annuali del ciclo idrologico, e non consentono inoltre la soluzione di problemi a scala locale, come ad es. lo sfruttamento degli acquiferi, poiché non sono in grado di fornire soluzioni distribuite nello spazio e nel tempo. Di conseguenza, i risultati che si ottengono da tali analisi sono di tipo qualitativo e semi-quantitativo e di scarsa utilità per una efficace gestione e pianificazione della risorsa idrica.

Per quanto riguarda le acque sotterranee, la Direttiva Quadro sulle Acque 2000/60/CE (EU 2000; recepita in Italia dal testo Unico in materia ambientale) ha richiesto, oltre alla definizione e caratterizzazione dei corpi idrici sotterranei (avvenuta in Italia nel primo decennio del 2000), un potenziamento delle attività di monitoraggio dei livelli e dello stato chimico delle acque; questo a sua volta ha portato alla disponibilità di una notevole quantità di dati, unitamente alla disponibilità di serie temporali di numerose variabili idrologiche. Ad oggi il contenuto informativo di tali dati viene raramente sfruttato appieno, in quanto spesso tali dati sono analizzati con algoritmi molto semplici, che forniscono una visione limitata della dinamica dei sistemi idrologici. La disponibilità di strumenti ICT che svolgono analisi utilizzando sia la dimensione spaziale, sia quella temporale delle informazioni, potrebbe contribuire a sfruttare il contenuto informativo di tali dati e ad ottenere una migliore comprensione del comportamento dei corpi idrici sotterranei.

Benché l'utilizzo dei Sistemi Informativi Geografici (GIS) nella gestione della risorsa idrica sia ormai ben standardizzato (cfr. le linee guida della Common Implementation Strategy 9 e 22 per l'applicazione della Direttiva Quadro sulle Acque; Ludovici e Toniutti 2007), in molti casi l'uso delle tecnologie informatiche è limitato ad azioni di base (come ad es. la produzione di cartografie o la compilazione di database spaziali). Questo costituisce un limite alle sfide e ai problemi posti oggigiorno dalla necessità di gestione della risorsa idrica.

Il valore dell'utilizzo di tecnologie avanzate (spesso definite "complesse" nei documenti legati alla WFD) è spesso limitato dal fatto che solo gli operatori e professionisti ben formati siano coinvolti nell'analisi e nella successiva presentazione dei risultati agli attori coinvolti in un determinato problema di gestione della risorsa. Di conseguenza, questi ultimi non sono attivamente coinvolti, ad esempio, nello sviluppo di modelli numerici e devono porre affidamento e fiducia in chi ha eseguito l'analisi (Refsgaard et al. 2010). In questo senso, la mancanza di un approccio partecipato per l'utilizzo di strumenti ICT avanzati, a supporto della formazione di un processo decisionale basato su evidenze di tipo scientifico, costituisce un limite all'applicazione delle direttive relative all'acqua. Da un punto di vista tecnico e sociale, ciò provoca una minore efficienza nel coordinamento tra personale tecnico/ scientifico, decisori e vari soggetti coinvolti nel monitoraggio e gestione della risorsa idrica.

Per superare questo approccio puramente tecnico nell'affrontare questioni molto complesse e con una grande varietà di stakeholder, è utile introdurre un approccio partecipato, che coinvolga stakeholder e specialisti tecnici del settore. In tale ottica, gli aspetti non tecnici dei sistemi idrologici (quali ad es. temi sociali, politici e giuridici) sono riconosciuti come rilevanti, se non fondamentali, per raggiungere decisioni condivise che vanno ad integrarsi alle questioni più tecniche $\mathrm{e}$ fisico/matematiche (Castelletti et al. 2007).

Le tecniche di simulazione modellistica e gli strumenti per l'analisi dei dati geospaziali possono contribuire alla corretta gestione dell'utilizzo congiunto di acque superficiali e sotterranee, anche per evitare conflitti tra i diversi utenti e la manutenzione degli agroecosistemi (Rossetto et al. 2013). Come tale, lo sviluppo di strumenti software innovativi per la gestione delle risorse idriche è fondamentale, anche in vista di una corretta applicazione della direttiva quadro EU sull'acqua (EU, 2000) e di altre direttive relative all'acqua.

FREEWAT (FREE and open source software tools for WATer resource management; Rossetto et al. 2015) è un progetto HORIZON 2020, finanziato dalla Commissione Europea, volto a promuovere l'applicazione delle direttive comunitarie relative all'acqua mediante: i) lo sviluppo di una piattaforma dedicata ed open source integrata in GIS per la pianificazione e la gestione delle risorse idriche superficiali e sotterranee, ii) ampie attività di formazione e infine iii) l'applicazione della piattaforma sviluppata a 14 casi di studio, nel contesto di un approccio partecipato appositamente sviluppato. Il Consorzio FREEWAT è costituito da 19 partners di 11 paesi EU (Italia, Germania, Spagna, Francia, Grecia, Estonia, Romania, Malta, Repubblica Ceca, Slovenia, Olanda), Svizzera, Turchia e Ucraina. L'UNESCO - International Hydrological Programme completa il Consorzio.

Questo contributo intende presentare la piattaforma FREEWAT e discuterne la sua applicazione.

\section{Materiali e metodi}

La struttura complessiva del progetto FREEWAT è basata su: i) il coordinamento di precedenti ricerche finanziate a livello nazionale ed europeo; ii) sostenere la diffusione della piattaforma FREEWAT attraverso percorsi di formazione specifica e una serie applicazioni a casi di studio; iii) sperimentare l'applicazione di FREEWAT in un approccio partecipativo innovativo, che vada a riunire sia il personale tecnico, sia le parti interessate nello sviluppo e nella simulazione di scenari per l'applicazione di politiche per la gestione della risorsa idrica; iv) comunicare le attività e i risultati di FREEWAT a livello internazionale al fine di promuovere la sua applicazione. Questi quattro elementi hanno guidato il flusso di lavoro del progetto.

Lo sviluppo della piattaforma FREEWAT (Fig. 1) è stato supportato dal risultato di un sondaggio condotto nella prima 


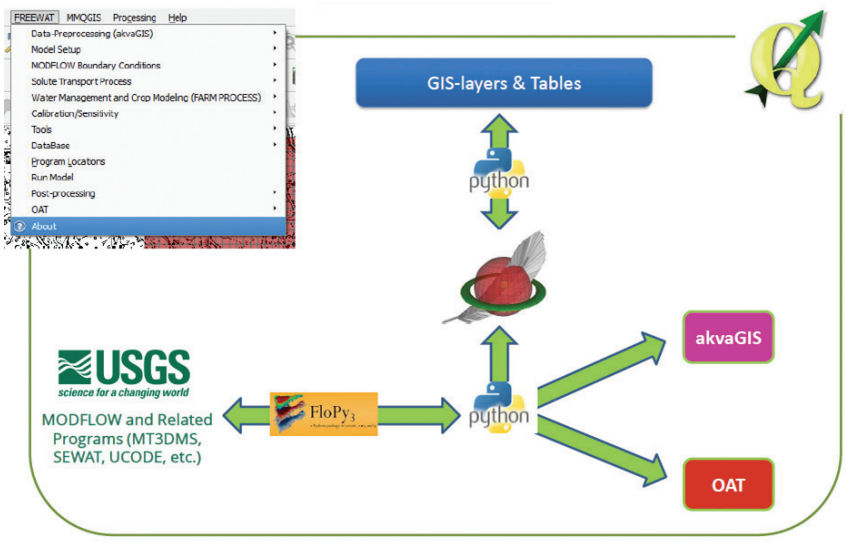

Fig. 1 - Simplified scheme of FREEWAT architecture (De Filippis et al. 2017).

Fig. 1 - Schema semplificato dell'architettura della piattaforma FREEWAT (De Filippis et al. 2017).

fase del progetto per elaborare una matrice di valutazione, sulla base della quale identificare i codici/moduli da integrare nella piattaforma stessa. Questo è stato fatto andando ad intersecare le esigenze/priorità relative alle problematiche di gestione delle risorse idriche, con la disponibilità di strumenti software per affrontare tali problematiche. I questionari di cui sopra sono stati distribuiti a 14 partner del progetto FREEWAT e a numerosi stakeholder (istituti di ricerca, autorità governative, aziende di consulenza geoambientali, autorità di distretto idrografico) nei paesi dell'UE e non solo. Le priorità identificate si riferiscono alla gestione dell'acqua in ambiente rurale (compresa la gestione delle sostanze agrochimiche) e ad una gestione sostenibile delle acque sotterranee in termini di qualità e quantità delle stesse. Diverse necessità sono poi legate direttamente o indirettamente all'uso congiunto delle acque superficiali e sotterranee, e alle interazioni tra corpi idrici superficiali e sotterranei. Le necessità e le priorità sono state quindi collegate anche alle direttive e ai relativi regolamenti UE. Durante lo sviluppo della piattaforma FREEWAT si è perciò cercato di dare risposta alle priorità identificate attraverso la matrice di valutazione.

La piattaforma FREEWAT è oggi un plugin per il GIS desktop QGIS (QGIS Development Team 2009), che consente di accoppiare la potenza degli strumenti di geo-elaborazione e post-elaborazione tipici dei software GIS per l'analisi dei dati spaziali, a quella dei modelli numerici per la simulazione di numerosi processi idrologici (beneficiando di codici sviluppati dallo United States Geological Survey - USGS, figura 1). Come tale, la piattaforma FREEWAT è concepita con una struttura modulare, in cui diversi codici per la simulazione del ciclo idrologico (con focus prevalente sull'analisi del flusso delle acque sotterranee) e processi idrochimici sono integrati nel desktop QGIS (Fig. 2). I dati di input e output sono gestiti tramite il database spaziale SpatiaLite (SpatiaLite Development Team 2011). Elemento centrale nello sviluppo di questa piattaforma è l'utilizzo di codici esclusivamente open source.

Gli strumenti ad oggi implementati nella piattaforma FREEWAT possono essere suddivisi in due grandi classi. Nella prima includiamo gli strumenti per l'analisi dei dati idrochimici e idrogeologici (modulo akvaGIS) e gli strumenti per il pre-processamento dei dati e per l'analisi delle serie temporali (modulo OAT). La seconda classe è costituita da una serie di strumenti per l'implementazione del modello numerico e include: (i) codici della famiglia MODFLOW USGS, che sono prevalentemente dedicati alla gestione delle acque sotterranee; (ii) un modulo per la simulazione della crescita della pianta e per la stima della resa colturale (Crop Growth Module - CGM), appartenente alla famiglia di modelli EPIC (Williams et al. 1989; Williams and Singh 1995; Gassman et al. 2005, 2007, 2009); (iii) strumenti di processing sviluppati per trasferire agevolmente i valori di alcune variabili (ad es., conducibilità idraulica) da layer GIS raster/vettoriali a celle della griglia. Di seguito si riporta il dettaglio degli strumenti disponibili.

Il modulo akvaGIS offre diversi strumenti per l'analisi e l'interpretazione dei dati idrochimici e idrogeologici. Attraverso questo modulo è possibile preparare grafici

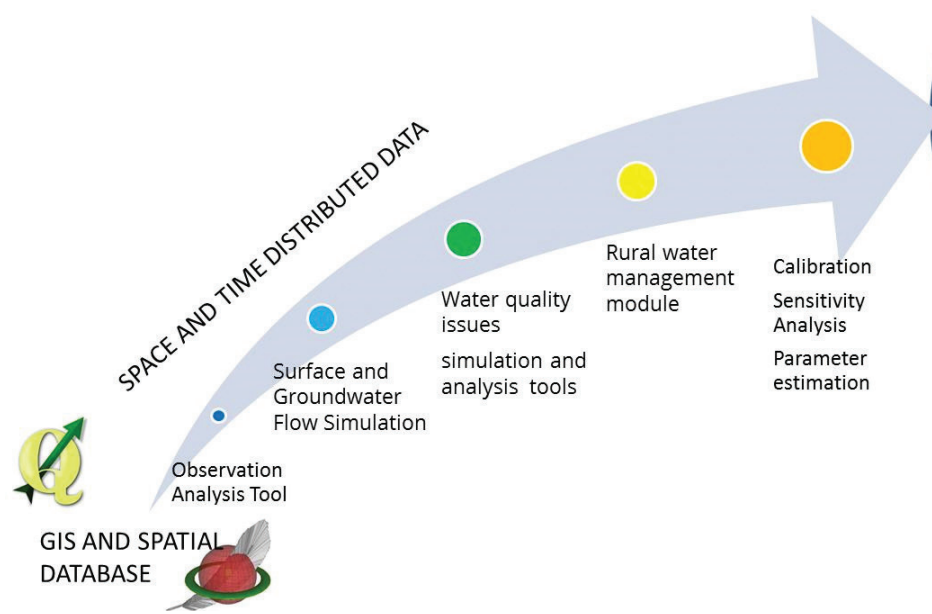

Fig. 2 - Overview of the tools integrated in FREEWAT (De Filippis et al. 2017).

Fig. 2 - Panoramica dei diversi strumenti integrati in FREEWAT (De Filippis et al. 2017). 
e realizzare analisi statistiche su dati idrochimici per la valutazione della qualità delle acque sotterranee, interpretare dati idrogeologici e generare mappe tematiche per la costruzione di modelli concettuali

Lo strumento Observation Analysis Tool (OAT) fornisce all'Utente funzionalità avanzate per l'analisi delle serie temporali, in vista anche di una crescente diffusione delle reti di monitoraggio, on-line e in tempo reale. OAT è progettato per facilitare l'importazione, l'analisi e la visualizzazione dei dati della serie temporale e l'utilizzo di questi dati per supportare la costruzione di modelli numerici e la loro calibrazione.

$\mathrm{La}$ simulazione del flusso delle acque sotterranee può essere effettuata utilizzando MODFLOW-2005 (Harbaugh 2005), un codice distribuito, fisicamente basato, sviluppato da USGS. Il flusso associato ai pozzi, la ricarica efficace, l'evapotraspirazione, i dreni e i corpi idrici superficiali possono essere simulati attraverso specifici pacchetti MODFLOW (CHD, WEL, MNW, RCH, RIV, LAK, DRN, GHB, EVT, $U Z F$, SFR; per ulteriori dettagli si faccia riferimento a Harbaugh 2005).

In FREEWAT, un modello idrologico può essere accoppiato con uno o più modelli di trasporto dei soluti, per simulare il trasporto advettivo-dispersivo multi-specie, nella zona satura, usando MT3DMS (Zheng and Wang 1999). La simulazione del trasporto di soluti 1D all'interno della zona insatura è possibile utilizzando uno tra i seguenti strumenti:

- il modulo USB (Unsaturated Solute Balance), per stimare la quantità di contaminante che, rilasciato al piano campagna, percola attraverso la zona insatura guidato da un flusso verticale puramente advettivo e raggiunge la superficie piezometrica, dove va a costituire una sorgente a concentrazione costante per la zona satura, alla quale viene applicato MT3DMS;

- il codice MT3D-USGS (Bedekar et al. 2016) per la simulazione del flusso advettivo-dispersivo nella zona insatura.

L'integrazione in FREEWAT del codice SEAWAT (Langevin et al. 2007) consente di simulare i flussi dipendenti dalla viscosità e dalla densità. Ciò permette di affrontare la simulazione di processi di intrusione di acqua di mare o la valutazione delle risorse geotermiche a bassa e media entalpia.

La gestione delle risorse idriche è realizzata integrando l'ultima versione del codice MODFLOW, MODFLOWOWHM (One-Water Hydrologic flow Model, Hanson et al. 2014), all'interno del quale il modulo Farm Process consente di simulare dinamicamente le componenti di domanda e di fornitura dell'uso dell'acqua per delle sotto-regioni del modello in cui si svolgono attività idro-esigenti, come quelle legate all'agricoltura. Viene così ottenuto un modello idrologico integrato e accoppiato, che permette la stima delle possibili allocazioni idriche derivanti dall'uso congiunto di acque superficiali e sotterranee. La gestione delle risorse in ambiente rurale può essere anche eseguita tenendo conto di eventuali norme per la regolarizzazione dell'approvvigionamento idrico.

UCODE_2014 (Poeter et al. 2014) è integrato per eseguire analisi di sensibilità e stima dei parametri, con l'obiettivo di migliorare la calibrazione dei modelli, riducendo il divario tra carico idraulico e flussi simulati e i dati osservati. Per eseguire questa analisi, possono essere utilizzati diversi indicatori statistici, al fine di valutare la costruzione del modello e di selezionare i parametri da stimare utilizzando un metodo di regressione inverso basato sulla valutazione di una funzione obiettivo.

Lo sviluppo della piattaforma ha incluso l'applicazione sperimentale dei suddetti moduli di pre-processing e simulazione. A questo scopo sono stati preparati un set di tutorial e sei manuali Utente (oltre ad un Reference Manual) per diffondere l'utilizzo della piattaforma FREEWAT come software standard per la gestione delle acque sotterranee e delle relazioni con le acque superficiali. Il materiale disponibile comprende: 1) lezioni sull'applicazione di MODFLOW-2005 per simulare la dinamica delle acque sotterranee, 2) lezioni che presentano i concetti teorici alla base del codice MODFLOW, 3) esercizi e presentazioni per ciascuno dei moduli presentati. Nel periodo 2016-2017 sono stati formati all'utilizzo della piattaforma FREEWAT circa 1100 Utenti a scala globale in circa 50 Paesi, provenienti dal mondo accademico, delle autorità idriche, e da aziende di consulenza, in circa 60 corsi dedicati.

La piattaforma FREEWAT è stata applicata a quattordici casi di studio in Paesi dell'UE e non UE, per affrontare questioni specifiche in materia di gestione delle risorse idriche. Tali casi di studio possono essere suddivisi in due cluster (Figura 3):

- nove casi (otto in Paesi dell'Unione Europea e uno in Svizzera) si concentrano specificamente sull'applicazione a problematiche legate alla direttiva quadro delle acque, alla direttiva sulle acque sotterranee e ad altre direttive sul tema. Il collegamento all'applicazione delle direttive comunitarie in materia di acque è presente, ad esempio, mediante la verifica della fattibilità delle misure previste dai Programmi di Misure che ogni autorità di bacino/ distretto idrografico ha fissato per raggiungere gli obiettivi posti dalla direttiva quadro sulle acque. Tra questi, vengono trattati i seguenti temi: processi di intrusione delle acque di mare, allocazione della risorsa sotterranea per prelievi idropotabili, gestione di corpi idrici transfrontalieri e/o ecosistemi dipendenti dalle acque sotterranee. Questo numero include la presentazione di due di questi casi studio relativi alla gestione della risorsa idrica sotterranea nella piana di Vrbanski, in Slovenia, e nella piana costiera di Follonica-Scarlino, in Italia;

- cinque casi di studio (due in Paesi dell'UE, uno in Ucraina, uno in Turchia e uno in Africa) sono dedicati ad affrontare questioni relative alla gestione delle risorse idriche in ambiente rurale, ai sensi delle norme EU e/o nazionali/locali. I problemi trattati in questi casi di studio riguardano, ad es., la riduzione del consumo di acqua nelle aree irrigue e/o dell'inquinamento da nitrati delle acque sotterranee. Questo numero include la presentazione di due di questi casi studio relativi alla gestione della risorsa idrica sotterranea nel bacino di Palas, in Turchia, e nel bacino di Bakumivka, in Ucraina. 

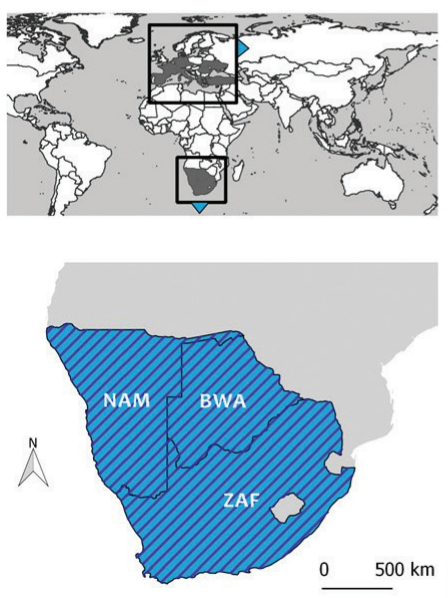

Implementation of the Water Framework Directive

Rural water management Both of the above categories

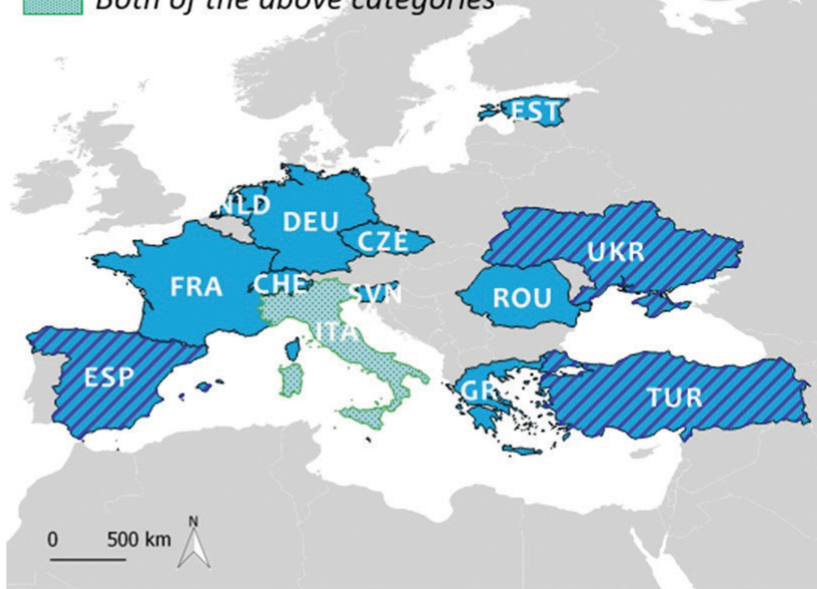

Fig. 3 - Main topics treated in 14 case studies for the application of the FREEWAT platform (De Filippis et. al 2017). This volume includes presentation of the following case studies: $V r$ banski Plato, in Slovenia, the coastal plain of Follonica-Scarlino, in Italy, Palas basin, in Turkey, Bakumivka basin, in Ukraine.

Fig. 3 - Le principali tematiche trattate nei 14 casi di studio per l'applicazione della piattaforma FREEWAT (De Filippis et al. 2017). Questo numero include la presentazione dei seguenti casi di studio: piana di Vrbanski, in Slovenia, piana costiera di Follonica-Scarlino, in Italia, bacino di Palas, in Turchia, bacino di Bakumivka, in Ucraina.
Per ogni caso di studio, la parte tecnica è stata svolta contemporaneamente ad una serie di incontri (Focus Group, FG) in cui si è testata l'efficacia dell'approccio partecipato nell'uso di strumenti ICT per la gestione della risorsa idrica. Questo ha costituito un punto fondamentale dell'intero progetto: i casi di studio sono stati condotti in modo innovativo e partecipato, coinvolgendo il personale tecnico e i principali stakeholder nella gestione della risorsa idrica. Detti stakeholder sono stati coinvolti nell'implementazione dei modelli numerici a partire dalla scelta dei dati, nella definizione di scenari, fino alla discussione dei risultati. Nei FG si è discusso delle conseguenze socioeconomiche e ambientali degli scenari analizzati. Questo approccio ha permesso, nei casi in esame, di accrescere la credibilità nei dati, nei risultati e nei modelli usati per testare la validità delle azioni previste.

\section{Conclusioni}

Lo sviluppo e distribuzione di una suite software open source e di pubblico dominio, integrata in GIS, che include anche una serie di codici numerici per la simulazione di numerosi processi idrologici, può costituire un avanzamento nella gestione delle risorse idriche. Le attività svolte hanno permesso di affrontare il tema dell'applicazione estesa di strumenti e tecnologie dell'ICT open source, in particolare dei modelli numerici. Si sono resi così disponibili strumenti ICT per l'analisi dell'uso congiunto delle acque superficiali e delle acque sotterranee, degli impatti connessi all'uso del suolo e del cambiamento climatico sulle risorse idriche. In tal modo, si offre la possibilità di modificare l'approccio per la definizione dei bilanci idrici, passando da bilanci calcolati annualmente come base per le decisioni, a bilanci distribuiti nello spazio e sulla base dei quali poter fare considerazioni anche nel tempo. In questo modo è possibile anche valorizzare un utilizzo efficace dei dati derivanti dalle attività di monitoraggio richieste dalla WFD. Infine si è proposto l'approccio partecipato non solo nella fase di discussione dei risultati, a valle di un lavoro/ studio con aspetti fortemente tecnici (come quello richiesto $\mathrm{da}$ analisi modellistiche), ma anche in tutte le fasi dell'analisi per affrontare questioni molto complesse relative alla gestione della risorsa idrica che vedono la partecipazione di una grande varietà di stakeholder.

La piattaforma FREEWAT è stata progettata per soddisfare le necessità di autorità idriche e imprese pubbliche/private nel costruire una rappresentazione altamente informativa e dinamica dei sistemi idrologici, tenendo conto della grande quantità di dati attualmente disponibili. Ciò con il fine ultimo di sostenere adeguatamente i risultati della ricerca scientifica per promuovere la loro reale applicazione di scala, replicabilità e assunzione da parte dei responsabili politici e delle autorità idriche.

La piattaforma FREEWAT unisce la potenza degli strumenti GIS di elaborazione e post-elaborazione per l'analisi dei dati spaziali (inclusa la gestione e la visualizzazione dei risultati delle elaborazioni effettuate), a quella dei software di simulazione. In questo modo si evita l'utilizzo di software di terze parti e, ad esempio, è possibile prevenire errori legati alla ridondanza dei dati. I decisori possono dunque trarre beneficio dall' applicazione della piattaforma FREEWAT, seguendo l'approccio partecipato proposto nel progetto.

Ringraziamenti: Questo documento è presentato nell'ambito del progetto FREEWAT, che ha ricevuto finanziamenti dal programma di ricerca e innovazione dell'Unione Europea Horizon 2020 nell'ambito del Contratto di sovvenzione n.642224.

Il contenuto di questo documento riflette solo le opinioni degli autori e l'Unione Europea non è responsabile per qualsiasi utilizzo che possa essere fatto delle informazioni in esso contenute. 


\section{BIBLIOGRAFIA}

Bedekar V, Morway ED, Langevin CD, Tonkin M (2016) MT3DUSGS version 1: A U.S. Geological Survey release of MT3DMS updated with new and expanded transport capabilities for use with MODFLOW. U.S. Geological Survey, Techniques and Methods 6-A53, 69 pp.

Castelletti A, De Rigo D, Rizzoli AE, Soncini-Sessa R, Weber E (2007) Neuro-dynamic programming for designing water reservoir network management policies. Control Engineering Practice 15(8):1031-1038.

De Filippis G, Borsi I, Foglia L, Cannata M, Velasco Mansilla V, Vasquez-Suñe E, Ghetta M, Rossetto R (2017) Software tools for sustainable water resources management: the GIS-integrated FREEWAT platform. Rend. Online Soc. Geol. It. 42:59-61. doi:10.3301/ROL.2017.14

EU (2000) Directive 2000/60/EC of the European Parliament and of the Council establishing a framework for the Community action in the field of water policy. Official Journal (OJ L 327) on 22 December 2000.

Gassman PW, Williams JR, Benson VW, Izaurralde RC, Hauck LM, Jones CA, Atwood JD, Kiniry JR, Flowers JD (2005) Historical development and applications of the EPIC and APEX models. Center for Agricultural and Rural Development, Iowa State University Ames.

Gassman PW, Reyes MR, Green CH, Arnold JG (2007) The soil and water assessment tool: historical development, applications, and future research directions.

Gassman PW, Williams JR, Wang X, Saleh A, Osei E, Hauck L, Izaurralde C, Flowers J (2009) The Agricultural Policy Environmental Extender (APEX) model: An emerging tool for landscape and watershed environmental analyses. Center for Agricultural and Rural Development (CARD) Publications, Ames.

Hanson RT, Boyce SE, Schmid W, Hughes JD, Mehl SM, Leake SA, Maddock T, Niswonger RG (2014) One-Water Hydrologic Flow Model (MODFLOW-OWHM). U.S. Geological Survey, Techniques and Methods 6-A51, $134 \mathrm{pp}$.

Harbaugh AW (2005) MODFLOW-2005, The U.S. Geological Survey Modular Ground-Water Model - the Ground-Water Flow Process. U.S. Geological Survey, Techniques and Methods 6-A16, 253 p.

Langevin CD, Thorne DT Jr, Dausman AM, Sukop MC, Weixing G (2007) SEAWAT Version 4: A Computer Program for Simulation of Multi-Species Solute and Heat Transport. U.S. Geological Survey Techniques and Methods 6-A22, 39 pp.
Ludovici AA, Toniutti N (2007) Strategia Comune di applicazione della Direttiva Quadro Acque (2000/60/EC), Traduzione del documento guida n. 1 ECONOMIA ED AMBIENTE - Le sfide nell'applicazione della Direttiva Quadro Acque. Novembre 2007 - WWF Italia Onlus. "Common strategy for the application of the water Directive 2000/60/EC, Translation of document n. 1 ECONOMY AND ENVIRONMENT - challenges in the application of the water Directive 2000/60/EC".

PoeterEP, Hill MC, Lu D, Tiedeman CR, Mehl S(2014)UCODE_2014, with new capabilities to define parameters unique to predictions, calculate weights using simulated values, estimate parameters with SVD, evaluate uncertainty with MCMC, and more. Integrated Groundwater Modeling Center Report Number GWMI 2014-02.

QGIS Development Team (2009) QGIS Geographic Information System. Open Source Geospatial Foundation Project. http://qgis.osgeo.org

Refsgaard JC, Storm B, Clausen T (2010) Système Hydrologique Europeén (SHE): review and perspectives after 30 years development in distributed physically-based hydrological modelling. Hydrology Research 41(5):355-377.

Rossetto R, Borsi I, Schifani C, Bonari E, Mogorovich P, Primicerio M (2013) SID\&GRID: Integrating hydrological modeling in GIS environment. Rendiconti Online Società Geologica Italiana 24:282283.

Rossetto R, Borsi I, Foglia L (2015) FREEWAT: FREE and open source software tools for WATer resource management. Rendiconti Online Società Geologica Italiana 35:252-255. doi: 10.3301/ ROL.2015.113

SpatiaLite Development Team (2011) The Gaia-SINS federated projects home-page. http://www.gaia-gis.it/gaia-sins/

Williams JR, Singh VP (1995) The EPIC model, In: Computer models of watershed hydrology, pp. 909-1000, V.J. Singh (Ed.), Water Resource Publications, Highlands Ranch, CO.

Williams JR, Jones CA, Kiniry JR, Spanel DA (1989) The Epic Crop Growth-Model. Transactions of the Asae 32:497-511.

Zheng C, Wang PP (1999) MT3DMS, A modular three-dimensional multi-species transport model for simulation of advection, dispersion and chemical reactions of contaminants in groundwater systems. U.S. Army Engineer Research and Development Center Contract Report SERDP-99-1, Vicksburg, MS, 202 pp. 